\title{
ASSESSMENT OF POLARIMETRIC VARIABILITY BY DISTANCE GEOMETRY FOR ENHANCED CLASSIFICATION OF OIL SLICKS USING SAR
}

\author{
Andrea Marinoni ${ }^{1}$, Martine M. Espeseth ${ }^{1}$, Paolo Gamba ${ }^{2}$, Camilla Brekke ${ }^{1}$, Torbjørn Eltoft ${ }^{1}$ \\ ${ }^{1}$ : Centre for Integrated Remote Sensing and Forecasting for Arctic Operations (CIRFA) \\ Dept. of Physics and Technology, UiT The Arctic University of Norway, Troms $\varnothing$, Norway. \\ E-mail: \{andrea.marinoni, martine.espeseth, camilla.brekke, torbjorn.eltoft\}@uit.no \\ 2: Dip. di Ingegneria Industriale e dell'Informazione, Università degli Studi di Pavia, Pavia, Italy. \\ E-mail:paolo.gamba@unipv.it
}

\begin{abstract}
In this paper, we introduce a new approach for investigation of polarimetric Synthetic Aperture Radar (PolSAR) images for oil slick analysis. Our method aims at enhancing discrimination of oil types by exploring the polarimetric features that can be produced by processing PolSAR scenes without dimensionality reduction. Taking advantage of a mixture description of the interactions among classes within the dataset and a characterization of their intra- and inter-class variability, our algorithm is able to quantify the areal coverage of different elements. These estimates can be used to hence improve classification. Experimental results on a PolSAR dataset acquired by unmanned aerial vehicle (UAV) on oil slicks in open water show the capacity of our method.
\end{abstract}

\section{INTRODUCTION}

Quad-Polarimetric (QP) SAR sensors provide multi-dimensional observations that have found many applications in Earth Observation (EO), including land cover classification, oil spill characterization, target detection and identification, and quantitative geophysical information extraction. One of the key advantages of QP-data is that they allow for target decomposition analysis, which provides information about the relative contributions from different scattering mechanisms [1]. A natural scene will in general consist of randomly distributed targets, resulting back-scattered signals, which are stochastic in nature. The target decomposition models are therefore based on second order statistics using the polarimetric covariance or coherence matrices. The fundamental assumption in all target decomposition methods is that the signal associated to each pixel in an image is the result of the interaction of multiple scattering mechanisms, and the aim of the analysis is to identify the relative power contribution of each of these mechanisms.

The UAVSAR scenes are courtesy of NASA/JPL-Caltech. This research is financed by CIRFA (RCN Grant no. 237906).
Target decomposition models are specific mixture models. In this paper, we introduce and explore a different type of mixture model for analyzing QP-data, which considers a general feature set extracted from polarimetric data (covariance matrix) as a linear mixture of contributions from certain primary ground cover classes (endmembers). The approach is inspired by the type of multidimensional analysis used in optical remote sensing to estimate fractional endmember abundances in an instantaneous field of view in a multispectral scene (see for instance [2]). The approach uses the complete high-dimensional data set, i.e. without dimensionality reduction, and aims at increasing classification performance by exploiting the characteristics of the data manifold induced by the polarimetric feature set. Here, we demonstrate the methodology by analyzing the classification performance in a marine oil spill experiment with four different oil spill types observed in a series of airborne QP-scenes. Specifically, the intrinsic variability within the features is used to improve the description of the scattering interactions occurring in oil slicks. In fact, due to wind and wave conditions the complexity of the scattering from the oil slicks and the ocean surface induce nonlinear effects on the feature sets (which may be implicitly highly correlated) [3]. This would typically lead to features characterized by data manifolds that might be nonconvex and nonlinear. In this case, pixel properties may not be properly described by a small amount of polarimetric features, i.e., dimensionality reduction and classic feature processing based on Euclidean geometry might lead to misclassification effects, as sparsity and nonlinearities could strongly degrade the characterization of the considered scenario [4]. The new processing scheme proposed here aims at avoiding information losses, as well as at recovering reliable characterization of the oil slicks by processing the classes feature sets and investigating their variability and nonlinearities.

The paper is organized as follows. Section 2 introduces the proposed method and its motivation. Section 3 reports details about the data considered in this study and the performance results. Finally, Section 4 delivers our final remarks 
and some ideas on future steps.

\section{METHODS}

Feature sets computed by processing intensity and phase of PolSAR sensed records can be considered as multidimensional signals. Hence, exploring the manifolds in high dimensional metric spaces induced by the feature set might help in avoiding undesired analysis effects (overfitting, noise amplification) and emphasizing hidden regularities among the records for understanding interactions among the elements [2, 4]. Specifically, it is possible to describe each feature set as a proper combination of the features that are associated with the extremal points (endmembers) of the aforesaid manifolds. Basically, the subspace induced by the endmembers contains all the other multidimensional records. In fact, endmembers represent a linear basis for the pixel feature set, with coordinates that are positive and sum to one. Hence, each feature set in the multidimensional feature space can be written as a combination of the endmembers' features. Then, if the endmember identify pure pixels (i.e., pixels that are not characterized by the combination of scatterings from multiple materials in the scene), the data manifold will form a simplex spanned by the endmembers in the multidimensional polarimetric space. Moreover, the barycentric coordinates would provide information on the actual composition of each pixel as a linear mixture proportion of the different materials, and classification can be therefore directly performed by inverting a linear optimization problem. In other terms, let $\underline{y}_{l}$ be the feature set of the $l$-th pixel in the $N$-dimensional feature space, i.e., $\underline{y}_{l}=\left[y_{l_{n}}\right]_{n=1, \ldots, N}, y_{l_{n}} \in \mathbb{R}$. Moreover, let us define $R$ as the number of endmembers, and $\underline{m}_{r}$ as the feature set of the $r$-th endmember. Thus, with the aforementioned observations on the manifold structure in mind, it is possible to write the following:

$$
\underline{y}_{l}=\sum_{r=1}^{R} a_{r l} \underline{m}_{r}
$$

where $a_{r l}$ identifies the proportion (fractional abundance) of the $r$-th endmember in the construction of the $l$-th feature set. As such, the $a$ parameters must fulfill the sum-to-one and nonnegativity constraints, i.e., $\sum_{r=1}^{R} a_{r l}=1 \forall l$, and $a_{r l} \geq 0$ $\forall(r, l)$, respectively. This approach hence requires identifying both the number and type of endmembers and their corresponding feature set in order to produce reliable estimates of those fractional abundances. Nonetheless, it is worth noting that the aforementioned scheme might not avoid characterization and classification errors. In fact, the use of fixed endmember feature sets does not take into account the variation in features caused by differential illumination conditions, spatial and temporal variability in the scene components, resulting in significant fraction estimate errors [4].
Indeed, the polarimetric variability in an image can be explained in terms of intra-class and inter-class variability [4]. In the first case, pixels belonging to the same class might show different polarimetric features as an effect of the different conditions of the imaging geometry and acquisition. In the second case, variations in features sets within the pixels arise from pixel-to-pixel variations in the fractional coverage. For each pixel, it may sometimes be more appropriate to recognize that a distribution of possible coverage may be derived for each class. The width of this distribution is a function of the degree of intra-class spectral variation (variability within the endmember class) present and will impact on the use of the sub-pixel classification output.

In order to properly address variability, we propose to consider each class in the linear mixture model in (1) as represented by the mean and by a variance-covariance matrix that captures the statistical variability around the mean. Indeed, inter-class variance is accounted by constructing a covariance matrix that varies around the selected endmember mean. Specifically, when we consider variability in (1), we expect the endmembers of each class to be drawn from a probability distribution defined by a probability density function (pdf) such as $\phi_{r}\left(\underline{m}_{r}\right)$. It is worth noting that it is possible to assume that endmember probability density functions for distinct classes are mutually independent. Hence, the probability density function of $\underline{y}_{l}$ would result from the convolution of the endmembers pdfs.

In order to extract information on these pdfs, our approach would require to follow two steps to consider nonlinear effects and endmember variability in the PolSAR data. First, we use distance geometry to characterize the volume spanned by the points in the dataset. Specifically, we aim at searching the simplex of largest volume in the data set. The data points that span this simplex then are the endmembers, under the assumption that a pure pixel is present in the data for every endmember. In order to address the nonlinear effects that can be showing up in the records, we use a distance geometry-based to detect the endmembers. In fact, it is possible to write the volume $\Omega$ of the subspace spanned by a set of $R$ points $\underline{z}_{1}, \ldots, \underline{z}_{R}$ in the multidimensional space according to the following:

$$
\Omega=\sqrt{\frac{\operatorname{det}[\underline{\underline{\Gamma}}]}{(-1)^{R} 2^{R-1}((R-1) !)^{2}}},
$$

where $\underline{\underline{\Gamma}}=\left[\Gamma_{i j}\right]_{(i, j) \in\{1, \ldots, R+1\}^{2}}$, and every $\Gamma_{i j}$ is set to the distance between $\underline{y}_{i}$ and $\underline{y}_{j}$ for $(i, j) \in\{1, \ldots, R\}^{2}$; to 0 for $(i, j)=(R+1, R+1)$; to 1 otherwise. The distances used in the volume calculation in (2) are Euclidean distances. Therefore, they might be not sufficient to directly describe the nonlinear effects of the PolSAR signals. On te other hand, if we use geodesic distances to characterize the data manifold that is spanned by the considered PolSAR records, we would be able to determine the effect of nonlinearities in feature sets. 
To this aim, we can use (2) together with a proper approximation of geodesic distances on a manifold, so that (2) can be used as an estimation of the geodesic volume one would find if the volume was to be measured along the data manifold.

A well-known data-driven approach for approximating geodesic distances on a manifold requires the construction of a nearest neighbor graph on the data, and measuring shortestpath distances along this graph [5]. To generate such a graph, we need to compute the Euclidean distance between any two points $\underline{y}_{i}$ and $\underline{y}_{j}$, and connect every point to the $\kappa$ nearest points, with $\kappa$ as a parameter of the algorithm. The weight of every edge is the corresponding Euclidean distance. Hence, the geodesic distance between two points is defined as the shortest-path distance along the weighted graph between these two points. The Dijkstra algorithm can be used to calculate the shortest-path distances from a point to all other points [6]. By defining distances in this way, these distances will approximate the true geodesic distances as measured along the surface of the data manifold.

Once we have built a weighted, symmetrical and connected $\kappa$-nearest neighbor graph on the data set, we can then start searching for the endmembers. Then, $R$ points are randomly selected as initial vertices. By means of the Dijkstra algorithm, the shortest-path distances from these $R$ vertices to all other points can be calculated. Thus, it is possible to compute the simplex volume when any vertex is replaced by a point that is randomly picked. If a larger simplex is found, the new vertex is kept, its distance to all other points with the Dijkstra algorithm is computed, and the definition of $\underline{\underline{\Gamma}}$ in (2) is updated. This search is performed until no larger simplex is found for any point. The outcome of this procedure is the definition of the endmembers $\underline{m}_{r}, r=1, \ldots, R$, among the feature sets of the considered dataset. At this stage, the abundances that would satisfy (1) can be obtained as $\underline{a}_{l}=\left(\mathcal{M}^{T} \mathcal{M}\right)^{-1}\left(\mathcal{M}^{T} \underline{y}_{l}-(\lambda / 2) \underline{1}\right)$, where $\underline{a}_{l}=\left[\underline{a}_{r l}\right]_{r=1, \ldots, R}$ and $\lambda$ is the Lagrangian multiplier to enforce the constraint on the abundances to be fulfilled. Moreover, $\mathcal{M}$ is a $N \times R$ matrix and the $r$-th column of $\mathcal{M}$ identifies the $r$-th endmember feature set. Finally, 1 is a vector of ones. However, in order to address variability of the records in the dataset, we ought to approximate the pdf of the endmembers within the dataset. Thus, we consider $K$ pixels around each endmember $\underline{m}_{r}$ in the multidimensional space, and we use them to approximate the statistical properties of each endmember. Specifically, let $\underline{\mu}_{r}$ and $\underline{\underline{C}}_{r}$ identify the mean vector and covariance matrix (computed across the $N$ polarimetric features) of the $r$-th endmember, respectively.

When no assumptions on the general pdf of the feature sets of the different classes can be drawn, we can always think that we have to face a linear optimization problem according to (1). Therefore, we must minimize the following objective function: $\mathcal{F}_{l}\left(\underline{a}_{l}, \lambda\right)=\mathbb{E}\left[\underline{\xi}^{T} \underline{\xi}\right]+\lambda\left(\sum_{r=1}^{R} a_{r l}-1\right)$, where $\underline{\xi}=\underline{y}_{l}-\sum_{r=1}^{R} a_{r l} \underline{m}_{r}$. Recalling the definition of $\underline{\mu}_{r}$ and $\underline{\underline{C}}_{r}$, and setting $\underline{\underline{D}}$ as the diagonal matrix of the traces of $\underline{\underline{C}} r$, then $\mathcal{F}_{l}\left(\underline{a}_{l}, \lambda\right)=\left(\underline{y}_{l}-\underline{\underline{\mu}}\right)^{T}\left(\underline{y}_{l}-\underline{\underline{\mu}}\right)+\underline{a}_{l}^{T} \underline{\underline{D} a_{l}}+\lambda\left(\underline{a}_{l}^{T} \underline{1}-\underline{1}\right)$, where $\underline{\underline{\mu}}=\left[\underline{\mu}_{r}\right]_{r=1, \ldots, R}$. In order to minimize $\mathcal{F}_{l}\left(\underline{a}_{l}, \lambda\right)$, we must

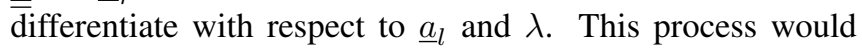
lead to $2 \underline{\mu}^{T} \underline{\mu} \underline{a}_{l}-2 \underline{\mu}^{T} \underline{y}_{l}+2 \underline{\underline{D} a_{l}}+\lambda \underline{1}=0$, so that $\underline{a}_{l}$ can be written as follows:

$$
\underline{a}_{l}=\left(\underline{\underline{\mu}}^{T} \underline{\underline{\mu}}+\underline{\underline{D}}\right)^{-1}(\underline{\underline{\mu}} \underline{\underline{y}}-\lambda \underline{1} / 2)
$$

Therefore, the variability of the endmembers distribution is taken into account by means of the mean and covariance of each endmember set.

\section{EXPERIMENTAL RESULTS}

The methods are tested on 21 L-band Uninhabited Aerial Vehicle Synthetic Aperture Radar (UAVSAR) scenes from the NOrwegian Radar oil Spill Experiment (NORSE2015) during the oil-on-water exercise in 2015. The scenes cover four substances, three emulsions with 40\% (E40), 60\% (E60), 80\% (E80) oil, and one plant oil (PO) for simulation of biogenic slicks. The four substances are masked out the same manner as in [3]. The reader is referred to [3] for additional information about this exercise and [7] for information about the UAVSAR sensor. The UAVSAR monitored the slicks for approximate 8 hours from release. Three different look directions (headings) were used during the acquisitions [3], with headings of $7^{\circ}$ ( $\approx$ upwind) (10 scenes), $187^{\circ}(\approx$ downwind) (10 scenes), and $142^{\circ}(\approx$ downwind) ( 1 scene). Fig. 1 shows a subscene of one of the 21 UAVSAR scenes, with the four oil slicks indicated. The input to the investigated methods are 10 polarimetric features (see Table 1), which are calculated using a $15 \times 61$ averaging window.

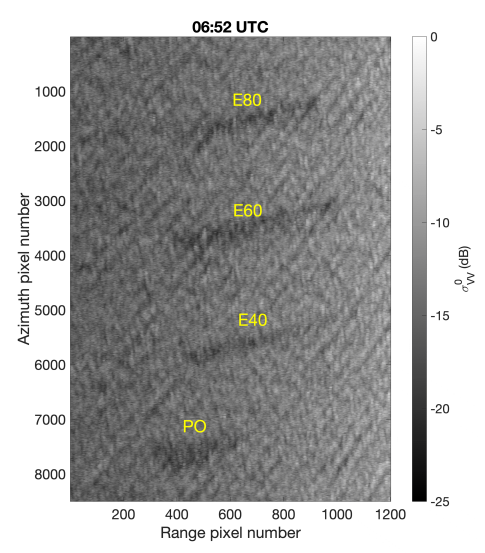

Fig. 1. VV-intensity $\left(\sigma_{V V}^{0}\right)$ in $\mathrm{dB}$ of one of the UAVSAR scenes with the oil slick types indicated.

The proposed method has been applied in order to understand the likelihood of the different substances to occur 
Table 1. Overview of the 10 investigated features. $S_{H H}$, $S_{V V}$, and $S_{H V}$ are the complex scattering vectors, and $\sigma_{H H}^{0}=\left\langle\left|S_{H H}\right|^{2}\right\rangle, \sigma_{V V}^{0}=\left\langle\left|S_{V V}\right|^{2}\right\rangle, \sigma_{H V}^{0}=\left\langle\left|S_{H V}\right|^{2}\right\rangle$ are the intensity products, where $\langle\cdot\rangle$ denotes spatial averaging with a $15 \times 61$ filter mask. $\mathrm{H}=$ horizontal polarization, $\mathrm{V}$ $=$ vertical polarization, the first subscript in $S$ or $\sigma^{0}$ is polarization on transmit, while the second subscript is polarization on receive.

\begin{tabular}{l|l}
\multicolumn{2}{c}{ Features } \\
\hline$\sigma_{V V}^{0}(d B)=10 \log 10\left(\sigma_{V V}^{0}\right)$ & $P D_{N}=\frac{\sigma_{V V}^{0}-\sigma_{H H}^{0}}{\sigma_{V V}^{0}+\sigma_{H H}^{0}}$ \\
$\sigma_{H H}^{0}(d B)=10 \log 10\left(\sigma_{H H}^{0}\right)$ & $\gamma_{H H / V V}=\sigma_{H H}^{0} / \sigma_{V V}^{0}$ \\
$\sigma_{H V}^{0}(d B)=10 \log 10\left(\sigma_{H V}^{0}\right)$ & $\gamma_{H V / H H}=\sigma_{H V}^{0} / \sigma_{H H}^{0}$ \\
$\mathrm{SPAN}=\sigma_{V V}^{0}+\sigma_{H V}^{0}+\sigma_{H H}^{0}$ & $r_{C O}=\Re\left(\left\langle S_{H H} S_{V V}^{\star}\right\rangle\right)$ \\
$P D=\sigma_{V V}^{0}-\sigma_{H H}^{0}$ & $\rho_{C O}=\frac{\left|\left\langle S_{H H} S_{V V}^{\star}\right\rangle\right|}{\sqrt{\left\langle\left|S_{H H}\right|^{2}\right\rangle\left\langle\left|S_{V V}\right|^{2}\right\rangle}}$
\end{tabular}

in every pixel. Following the notation in the previous Section, the number of endmember $R$ we considered has been set to 4 , while the number of neighbors $K$ for statistical approximation was set to 10 . Classification is then performed by majority voting rule. Fig. 2 shows the classification results for the different types of oil. Specifically, the radar plots report for the set of pixels that have been labeled as belonging to the four different classes (E80, E60, E40, and PO in Fig. 2(a) to (d), respectively) the classification estimates obtained by means of the algorithm proposed in this paper (blue line) and a classic inversion of the model in (1) without addressing endmember variability (orange line). For instance, when considering the E80-labeled pixels, we can notice that the proposed algorithm is able to correctly classify $80 \%$ of the pixels, while $10 \%$ of them were assigned to the E60 class (Fig. 2(a)). Therefore, it is possible to appreciate that the approach we propose is able to improve classification by taking into account the statistical distribution of the classes in the dataset consisting of 21 UAVSAR scenes. Hence, we can expect our method to increase understanding of complex PolSAR scenes, as it avoids nonlinear dimensionality reduction and therefore possible information losses.

\section{CONCLUSIONS}

In this paper, a new method for investigation of PolSAR images has been introduced. The approach we propose aims at improving classification by exploring the polarimetric features that can be produced by processing PolSAR scenes. Addressing inter- and intra-class variability within the records, the proposed architecture is able to enhance the classification performance and provide a better characterization of Earth surface. As this scheme is able to estimate the areal coverage of different classes on the considered region, it is expected that it can be used to deliver precise quantification of the different physical phenomena occurring on ground. Fur-

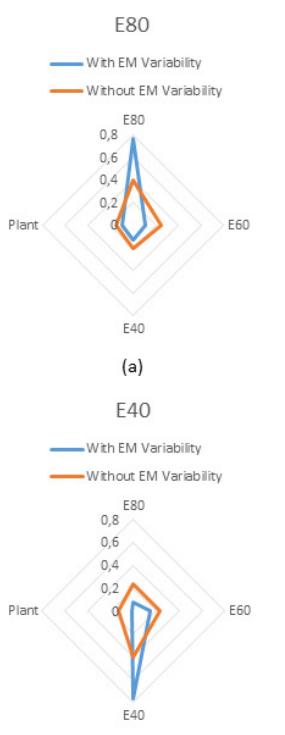

(c)

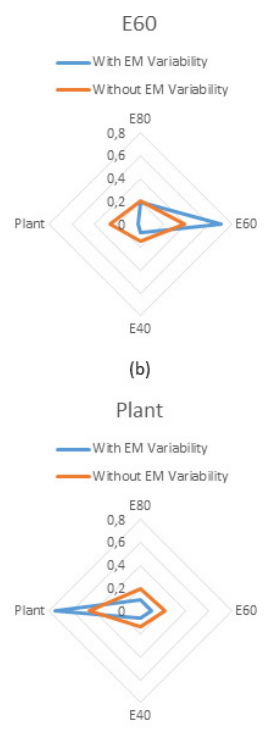

(d)
Fig. 2. Classification results for the different emulsions on the considered dataset

thermore, as no dimensionality reduction is employed, the proposed framework should minimize information losses in PolSAR image processing. These points will be properly discussed and evaluated in future works.

\section{REFERENCES}

[1] S.R. Cloude and E. Pottier. A review of target decomposition theorems in radar polarimetry. IEEE Trans. Geosci. Remote Sens., 34(2):498-518, 1996.

[2] A. Marinoni and P. Gamba. A novel approach for efficient $p$-linear hyperspectral unmixing. IEEE J. Select. Topics Sig. Proc., 9(6):1156-1168, Sept. 2015.

[3] S. Skrunes, C. Brekke, C. E. Jones, M. M. Espeseth, and B. Holt. Effect of wind direction and incidence angle on polarimetric SAR observations of slicked and unslicked sea surfaces. Remote Sens. Environ., 213:73 - 91, 2018.

[4] C. Collet, J. Chanussot, and K. Chehdi. Multivariate image processing. Wiley and sons, New York, NY, 2010.

[5] B. Song et al. Remotely sensed image classification using sparse representations of morphological attribute profiles. IEEE Trans. Geosci. Remote Sens., 52(8):51225136, 2014.

[6] E.W. Dijkstra. A note on two problems in connexion with graphs. Numerische Mathematik, 1:269-271, 1959.

[7] A. G. Fore et al. Uavsar Polarimetric Calibration. IEEE Trans. Geosci. Remote Sens., 53(6):3481-3491, June 2015. 